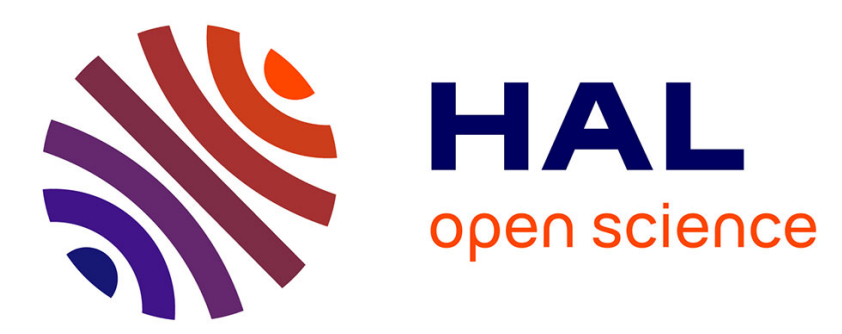

\title{
On the inversion of non symmetric sixth-order isotropic tensors and conditions of positiveness of third-order tensor valued quadratic functions
}

Vincent Monchiet, Guy Bonnet

\section{- To cite this version: \\ Vincent Monchiet, Guy Bonnet. On the inversion of non symmetric sixth-order isotropic tensors and conditions of positiveness of third-order tensor valued quadratic functions. Mechanics Research Communications, 2011, 38 (4), pp.326-329. 10.1016/j.mechrescom.2011.03.006 . hal-00687815}

\section{HAL Id: hal-00687815 https://hal.science/hal-00687815}

Submitted on 18 Apr 2012

HAL is a multi-disciplinary open access archive for the deposit and dissemination of scientific research documents, whether they are published or not. The documents may come from teaching and research institutions in France or abroad, or from public or private research centers.
L'archive ouverte pluridisciplinaire HAL, est destinée au dépôt et à la diffusion de documents scientifiques de niveau recherche, publiés ou non, émanant des établissements d'enseignement et de recherche français ou étrangers, des laboratoires publics ou privés. 


\title{
On the inversion of non symmetric sixth-order isotropic tensors and conditions of positiveness of third-order tensor valued quadratic functions
}

\author{
Vincent MONCHIET ${ }^{\mathrm{a}}$, Guy BONNET ${ }^{\mathrm{a}}$
}

a Universit Paris-Est, Laboratoire Modlisation et Simulation Multi Echelle, LMSME FRE3160 CNRS, 5 boulevard Descartes, 77454 Marne la Vallée Cedex, France

\begin{abstract}
In the present paper we propose new results concerning linear tensorial algebra for third-order and non symmetric isotropic sixth-order tensors in the most general case (i.e. having not the major and minor symmetries). Such tensors are used, for instance, in the theory of microstructured elastic media. A formalism based on an irreducible basis for isotropic sixth-order tensors is introduced, which is useful for performing the classical tensorial operations. Specially, a condensed expression for the product between two isotropic sixth-order tensors is provided, which allows the obtaining of a condition on these tensors for being invertible and a closed form expression of the inverse of such a tensor. Finally, the condition of positiveness of third-order tensor-valued quadratic functions is derived. For instance, such conditions are required for computing the elastic energy of microstructured media.
\end{abstract}

Key words: Sixth-Order Tensors, Third-Order Tensors, Isotropic Tensors, Microstructured Media

\section{Introduction}

Generalized continuum theories use tensors of-order $2,3 \ldots$ as variables as shown for instance by Toupin [8] or Mindlin [1], Mindlin and Eshel [2]. In these theories, the constitutive equations for elasticity take the form of a linear mapping between tensors of degree $2,3 \ldots$ which involves the introduction of elastic tensors whose degrees are $4,5,6 \ldots$. The use of such high-order tensors raises a number of fundamental questions, specially

Email addresses: vincent.monchiet@univ-mlv.fr (Vincent MONCHIET), guy.bonnet@univ-mlv.fr (Guy BONNET).

Preprint submitted to Elsevier

5 April 2011 
concerning their inversion or the condition of positiveness when computing the elastic energy from these tensors.

Here we restrict our analysis to the theories which use third-order tensors. For instance, the third gradient theory uses the gradient of strain, $\kappa_{i j k}=\nabla_{k} \varepsilon_{i j}$ (see [2]) whereas the theory of microstructured media uses the gradient of microstrain $\chi_{i j k}=\nabla_{k} \psi_{i j}$ (see [1]). When an hypothesis of isotropy and centrosymmetry is used, the elastic constitutive equations for the both theories contain a linear relation between two third-order tensors, denoted $\boldsymbol{a}$ and $\boldsymbol{b}$, which can be put into the form:

$$
\boldsymbol{b}=\mathbb{A} \odot_{3} \boldsymbol{a}
$$

Where $\mathbb{A}$ is a constant sixth-order tensor, namely independent of $\boldsymbol{a}$. In (1), the symbol $\odot_{3}$ represents the triple contraction between two tensors $\boldsymbol{x}$ and $\boldsymbol{y}:\left[\boldsymbol{x} \odot_{3} \boldsymbol{y}\right]_{i . . j}=x_{i . . p q r} y_{p q r . . j}$. For instance, in (1), the components of $\boldsymbol{b}$ are $b_{i j k}=A_{i j k p q r} a_{p q r}$.

Two particular cases of (1) can be considered:

- (i) tensors $\boldsymbol{a}$ and $\boldsymbol{b}$ are symmetric with respect to their first two indices, namely: $a_{i j k}=a_{j i k}$ and $b_{i j k}=b_{j i k}$. It corresponds to the case of gradient elastic theory (since the strain is a symmetric tensor), $\boldsymbol{a} \equiv \nabla \boldsymbol{\varepsilon}$ and $\boldsymbol{b}$ is called hyperstress. It follows that $\boldsymbol{a}$ and $\boldsymbol{b}$ are defined by only 18 independent components. The sixth-order tensor $\mathbb{A}$ has then the following minor symmetries: $A_{i j k p q r}=A_{j i k p q r}$ and $A_{i j k p q r}=A_{i j k q p r}$.

- (ii) tensors $\boldsymbol{a}$ and $\boldsymbol{b}$ have no symmetry and are defined by 27 independent coefficient. It corresponds to the case of microstructured elastic media, $\boldsymbol{a} \equiv \nabla \boldsymbol{\psi}$ and $\boldsymbol{b}$ is called double stress. It follows that $A_{i j k p q r}$ has no more the minor symmetries.

In a recent paper [3], an irreducible basis for isotropic $2 n^{\text {th }}$-order tensors (with $n=$ $3,4, \ldots)$ having minor symmetries has been provided. It has been shown that the formalism introduced in this work is useful for doing the classical tensorial operations and specially the inversion of symmetric isotropic tensors of degree up to 8. However, the isotropic tensors considered in this study possessed the minor symmetries which restrict the domain of application of this formalism to the case of gradient theories. As it will be shown thereafter, a key to study the algebra is the decomposition between spherical and deviatoric parts. In this context, it is worthwhile to notice some studies which deal with the decomposition of third-order tensors and third-order tensor valued functions (see for instance Pennisi \& Trovato [4], Pennisi [5], Smyshlyaev \& Fleck [7], Smith [6]). However, all those studies dealt only with symmetric third-order tensors.

In the present paper, we aim at generalizing the results provided in [3] to the case of a sixth-order tensor which does not posses minor symmetries. A representation of such tensors in a basis constituted of 15 non symmetric isotropic tensors is provided in section 2. This basis is shown to be useful for deriving a closed form expression of the inverse of a non symmetric isotropic sixth-order tensor (namely having not the minor and the major symmetries). In section 3 , we derive the condition of positiveness of $\mathbb{A}$ in the case of tensors having the major symmetry (symmetry between the first three indices and the last three indices), which can be written:

$$
\forall \boldsymbol{a} \neq 0: \boldsymbol{a} \odot_{3} \mathbb{A} \odot_{3} \boldsymbol{a}>0
$$

Here, we deal with tensors having the major symmetries (but not the minor symmetries). Condition (2) is needed, for instance, for obtaining the condition of positiveness of the 
elastic energy of microstructured media.

\section{An irreducible basis for isotropic sixth-order tensors}

In [1], the following decomposition for an isotropic sixth-order tensor $\mathbb{A}$ is used:

$$
\mathbb{A}=\sum_{n=1}^{n=15} a_{n} \mathbb{T}_{n}
$$

where the components of tensors $\mathbb{T}_{n}$ for $n=1 . .15$ are given by:

$$
\begin{array}{lll}
{\left[\mathbb{T}_{1}\right]_{i j k p q r}=\delta_{i p} \delta_{j q} \delta_{k r},} & {\left[\mathbb{T}_{2}\right]_{i j k p q r}=\delta_{i r} \delta_{j p} \delta_{k q},} & {\left[\mathbb{T}_{3}\right]_{i j k p q r}=\delta_{i q} \delta_{j r} \delta_{k p}} \\
{\left[\mathbb{T}_{4}\right]_{i j k p q r}=\delta_{i r} \delta_{j q} \delta_{k p},} & {\left[\mathbb{T}_{5}\right]_{i j k p q r}=\delta_{i p} \delta_{j r} \delta_{k q},} & {\left[\mathbb{T}_{6}\right]_{i j k p q r}=\delta_{i q} \delta_{j p} \delta_{k r}} \\
{\left[\mathbb{T}_{7}\right]_{i j k p q r}=\delta_{i j} \delta_{k r} \delta_{p q},} & {\left[\mathbb{T}_{8}\right]_{i j k p q r}=\delta_{i j} \delta_{k p} \delta_{q r},} & {\left[\mathbb{T}_{9}\right]_{i j k p q r}=\delta_{i j} \delta_{k q} \delta_{p r}} \\
{\left[\mathbb{T}_{10}\right]_{i j k p q r}=\delta_{i k} \delta_{j r} \delta_{p q},} & {\left[\mathbb{T}_{11}\right]_{i j k p q r}=\delta_{i k} \delta_{j p} \delta_{q r},} & {\left[\mathbb{T}_{12}\right]_{i j k p q r}=\delta_{i k} \delta_{j q} \delta_{p r}} \\
{\left[\mathbb{T}_{13}\right]_{i j k q q}=\delta_{j k} \delta_{i r} \delta_{p q},} & {\left[\mathbb{T}_{14}\right]_{i j k p q r}=\delta_{j k} \delta_{i p} \delta_{q r},} & {\left[\mathbb{T}_{15}\right]_{i j k p q}=\delta_{j k} \delta_{i q} \delta_{p r}}
\end{array}
$$

When a tensor $\mathbb{A}$ possesses the major symmetry, $A_{i j k p q r}=A_{\text {pqrijk }}$, the following conditions for the $a_{n}$ are required: $a_{2}=a_{3}, a_{8}=a_{13}, a_{9}=a_{10}, a_{11}=a_{15}$. In addition, the conditions for $\mathbb{A}$ to possess the minor symmetries are detailed in [3].

The 15 tensors $\mathbb{T}_{n}$ for $n=1 . .15$ constitute an irreducible basis for any isotropic sixthorder tensors. However, this basis appears to be not useful for obtaining the inverse of $\mathbb{A}$ since $\mathbb{T}_{n} \odot_{3} \mathbb{T}_{m} \neq 0$ whatever the values of $n$ and $m$. The use of basis $\mathbb{T}_{n}$ for the inversion of a sixth-order tensor leads then to a full linear system of dimension 15. For this reason, we propose a new basis denoted $\left(\mathbb{K}_{n}, \mathbb{J}_{m}\right)$ which is more convenient for inverting $\mathbb{A}$. This basis is constituted of sixth-order tensors denoted by $\mathbb{K}_{n}$ for $n=1 . .6$, which are:

$$
\begin{aligned}
\mathbb{K}_{1}= & \frac{1}{6}\left[\mathbb{T}_{1}+\mathbb{T}_{2}+\mathbb{T}_{3}-\mathbb{T}_{4}-\mathbb{T}_{5}-\mathbb{T}_{6}\right] \\
\mathbb{K}_{2}= & \frac{1}{6}\left[\mathbb{T}_{1}+\mathbb{T}_{2}+\mathbb{T}_{3}+\mathbb{T}_{4}+\mathbb{T}_{5}+\mathbb{T}_{6}\right] \\
& -\frac{1}{15}\left[\mathbb{T}_{7}+\mathbb{T}_{8}+\mathbb{T}_{9}+\mathbb{T}_{10}+\mathbb{T}_{11}+\mathbb{T}_{12}+\mathbb{T}_{13}+\mathbb{T}_{14}+\mathbb{T}_{15}\right] \\
\mathbb{K}_{3}= & \frac{1}{6}\left[\mathbb{T}_{10}+\mathbb{T}_{11}-\mathbb{T}_{13}-\mathbb{T}_{14}\right]+\frac{1}{3}\left[\mathbb{T}_{1}+\mathbb{T}_{4}+\mathbb{T}_{15}-\mathbb{T}_{3}-\mathbb{T}_{6}-\mathbb{T}_{12}\right] \\
\mathbb{K}_{4}= & \frac{1}{6}\left[\mathbb{T}_{11}+\mathbb{T}_{12}-\mathbb{T}_{14}-\mathbb{T}_{15}\right]+\frac{1}{3}\left[\mathbb{T}_{3}+\mathbb{T}_{5}+\mathbb{T}_{13}-\mathbb{T}_{2}-\mathbb{T}_{4}-\mathbb{T}_{10}\right] \\
\mathbb{K}_{5}= & \frac{1}{6}\left[\mathbb{T}_{7}+\mathbb{T}_{8}-\mathbb{T}_{13}-\mathbb{T}_{14}\right]+\frac{1}{3}\left[\mathbb{T}_{2}+\mathbb{T}_{5}+\mathbb{T}_{15}-\mathbb{T}_{3}-\mathbb{T}_{6}-\mathbb{T}_{9}\right] \\
\mathbb{K}_{6}= & \frac{1}{6}\left[\mathbb{T}_{8}+\mathbb{T}_{9}-\mathbb{T}_{14}-\mathbb{T}_{15}\right]+\frac{1}{3}\left[\mathbb{T}_{1}+\mathbb{T}_{6}+\mathbb{T}_{13}-\mathbb{T}_{2}-\mathbb{T}_{4}-\mathbb{T}_{7}\right]
\end{aligned}
$$

and 9 tensors denoted $\mathbb{J}_{m}$ for $m=1 . .9$ which are defined by: 


$$
\begin{array}{ll}
\mathbb{J}_{1}=\frac{1}{10}\left[4 \mathbb{T}_{14}-\mathbb{T}_{13}-\mathbb{T}_{15}\right], \quad \mathbb{J}_{2}=\frac{1}{10}\left[4 \mathbb{T}_{15}-\mathbb{T}_{14}-\mathbb{T}_{13}\right] \\
\mathbb{J}_{3}=\frac{1}{10}\left[4 \mathbb{T}_{13}-\mathbb{T}_{15}-\mathbb{T}_{14}\right], \quad \mathbb{J}_{4}=\frac{1}{10}\left[4 \mathbb{T}_{11}-\mathbb{T}_{10}-\mathbb{T}_{12}\right] \\
\mathbb{J}_{5}=\frac{1}{10}\left[4 \mathbb{T}_{12}-\mathbb{T}_{10}-\mathbb{T}_{11}\right], \quad \mathbb{J}_{6}=\frac{1}{10}\left[4 \mathbb{T}_{10}-\mathbb{T}_{11}-\mathbb{T}_{12}\right] \\
\mathbb{J}_{7}=\frac{1}{10}\left[4 \mathbb{T}_{8}-\mathbb{T}_{7}-\mathbb{T}_{9}\right], \quad \mathbb{J}_{8}=\frac{1}{10}\left[4 \mathbb{T}_{9}-\mathbb{T}_{7}-\mathbb{T}_{8}\right], \quad \mathbb{J}_{9}=\frac{1}{10}\left[4 \mathbb{T}_{7}-\mathbb{T}_{8}-\mathbb{T}_{9}\right]
\end{array}
$$

It can be shown that the $\mathbb{J}_{n}$ for $n=1 . .9$ and the $\mathbb{K}_{n}$ for $n=1 . .6$ have the property $\mathbb{K}_{n} \odot_{3} \mathbb{J}_{m}=\mathbb{J}_{m} \odot_{3} \mathbb{K}_{n}=0$ whatever the values of $n$ and $m$. The triple contraction between two elements taken from $\left(\mathbb{J}_{1}, \ldots, \mathbb{J}_{9}\right)$ are given in table 1 . The triple contraction between two elements taken from $\left(\mathbb{K}_{1}, \ldots, \mathbb{K}_{6}\right)$ are given in table 2 .

\begin{tabular}{|c|c|c|c|c|c|c|c|c|c|}
\hline$\odot_{3}$ & $\mathbb{J}_{1}$ & $\mathbb{J}_{2}$ & $\mathbb{J}_{3}$ & $\mathbb{J}_{4}$ & $\mathbb{J}_{5}$ & $\mathbb{J}_{6}$ & $\mathbb{J}_{7}$ & $\mathbb{J}_{8}$ & $\mathbb{J}_{9}$ \\
\hline $\mathbb{J}_{1}$ & $\mathbb{J}_{1}$ & $\mathbb{J}_{2}$ & $\mathbb{J}_{3}$ & 0 & 0 & 0 & 0 & 0 & 0 \\
\hline $\mathbb{J}_{2}$ & 0 & 0 & 0 & $\mathbb{J}_{1}$ & $\mathbb{J}_{2}$ & $\mathbb{J}_{3}$ & 0 & 0 & 0 \\
\hline $\mathbb{J}_{3}$ & 0 & 0 & 0 & 0 & 0 & 0 & $\mathbb{J}_{1}$ & $\mathbb{J}_{2}$ & $\mathbb{J}_{3}$ \\
\hline $\mathbb{J}_{4}$ & $\mathbb{J}_{4}$ & $\mathbb{J}_{5}$ & $\mathbb{J}_{6}$ & 0 & 0 & 0 & 0 & 0 & 0 \\
\hline $\mathbb{J}_{5}$ & 0 & 0 & 0 & $\mathbb{J}_{4}$ & $\mathbb{J}_{5}$ & $\mathbb{J}_{6}$ & 0 & 0 & 0 \\
\hline $\mathbb{J}_{6}$ & 0 & 0 & 0 & 0 & 0 & 0 & $\mathbb{J}_{4}$ & $\mathbb{J}_{5}$ & $\mathbb{J}_{6}$ \\
\hline $\mathbb{J}_{7}$ & $\mathbb{J}_{7}$ & $\mathbb{J}_{8}$ & $\mathbb{J}_{9}$ & 0 & 0 & 0 & 0 & 0 & 0 \\
\hline $\mathbb{J}_{8}$ & 0 & 0 & 0 & $\mathbb{J}_{7}$ & $\mathbb{J}_{8}$ & $\mathbb{J}_{9}$ & 0 & 0 & 0 \\
\hline $\mathbb{J}_{9}$ & 0 & 0 & 0 & 0 & 0 & 0 & $\mathbb{J}_{7}$ & $\mathbb{J}_{8}$ & $\mathbb{J}_{9}$ \\
\hline
\end{tabular}

\begin{tabular}{|c|c|c|c|c|c|c|}
\hline$\odot_{3}$ & $\mathbb{K}_{1}$ & $\mathbb{K}_{2}$ & $\mathbb{K}_{3}$ & $\mathbb{K}_{4}$ & $\mathbb{K}_{5}$ & $\mathbb{K}_{6}$ \\
\hline $\mathbb{K}_{1}$ & $\mathbb{K}_{1}$ & 0 & 0 & 0 & 0 & 0 \\
\hline $\mathbb{K}_{2}$ & 0 & $\mathbb{K}_{2}$ & 0 & 0 & 0 & 0 \\
\hline $\mathbb{K}_{3}$ & 0 & 0 & $\mathbb{K}_{3}$ & $\mathbb{K}_{4}$ & 0 & 0 \\
\hline $\mathbb{K}_{4}$ & 0 & 0 & 0 & 0 & $\mathbb{K}_{3}$ & $\mathbb{K}_{4}$ \\
\hline $\mathbb{K}_{5}$ & 0 & 0 & $\mathbb{K}_{5}$ & $\mathbb{K}_{6}$ & 0 & 0 \\
\hline $\mathbb{K}_{6}$ & 0 & 0 & 0 & 0 & $\mathbb{K}_{5}$ & $\mathbb{K}_{6}$ \\
\hline
\end{tabular}

Table 2: The triple contraction between the $\mathbb{K}_{n}$ for $n=1 . .6$

Table 1: The triple contraction between the

$$
\mathbb{J}_{n} \text { for } n=1 . .9
$$

Denoting by $\mathcal{E}_{6}$ the space of isotropic sixth-order tensors, the basis $\left(\mathbb{K}_{n}, \mathbb{J}_{m}\right)$ constitutes an irreducible basis for $\left(\mathcal{E}_{6}, \odot_{3}, \mathbb{I}\right)$ where $\mathbb{I}=\mathbb{T}_{1}$ is the identity for the composition $\odot_{3}$. Moreover, it can be observed that $\left(\mathcal{E}_{6}, \odot_{3}, \mathbb{I}\right)$ define a monoid, namely an algebraic structure with a single associative binary operation and an identity element.

Every tensor $\mathbb{A} \in \mathcal{E}_{6}$ is then decomposed as follows:

$$
\begin{aligned}
\mathbb{A}= & \alpha_{1} \mathbb{K}_{1}+\alpha_{2} \mathbb{K}_{2}+\alpha_{3} \mathbb{K}_{3}+\alpha_{4} \mathbb{K}_{4}+\alpha_{5} \mathbb{K}_{5}+\alpha_{6} \mathbb{K}_{6}+\alpha_{7} \mathbb{J}_{1}+\alpha_{8} \mathbb{J}_{2} \\
& +\alpha_{9} \mathbb{J}_{3}+\alpha_{10} \mathbb{J}_{4}+\alpha_{11} \mathbb{J}_{5}+\alpha_{12} \mathbb{J}_{6}+\alpha_{13} \mathbb{J}_{7}+\alpha_{14} \mathbb{J}_{8}+\alpha_{15} \mathbb{J}_{9}
\end{aligned}
$$

$\alpha_{1}, \ldots, \alpha_{15}$ are the components of $\mathbb{A}$ in the $\left(\mathbb{K}_{n}, \mathbb{J}_{m}\right)$ basis. The base change relations, giving coefficients $\alpha_{n}$ as function of the $a_{n}$ are provided in appendix $A$.

Denoting by $\mathcal{J}$ the sub-space of isotropic sixth-order tensors given by $\mathbb{A}=\alpha_{7} \mathbb{J}_{1}+$ $\ldots+\alpha_{15} \mathbb{J}_{9}\left(\right.$ or $\alpha_{1}=\alpha_{2}=\ldots=\alpha_{6}=0$ in $\left.(7)\right)$, it can be observed that $\left(\mathcal{J}, \odot_{3}, \mathbb{J}\right)$ define a submonoid, here $\mathbb{J}=\mathbb{J}_{1}+\mathbb{J}_{5}+\mathbb{J}_{9}$ is the unit element of $\mathcal{J}$ for the composition $\odot_{3}$. Similarly, we denote by $\mathcal{K}$ the sub-space of isotropic sixth-order tensors given by $\mathbb{A}=\alpha_{1} \mathbb{K}_{1}+\ldots+\alpha_{6} \mathbb{K}_{6}$. It can be observed that $\left(\mathcal{K}, \odot_{3}, \mathbb{K}\right)$ define a submonoid, $\mathbb{K}=$ 
$\mathbb{K}_{1}+\mathbb{K}_{2}+\mathbb{K}_{3}+\mathbb{K}_{6}$ being the unit element of $\mathcal{K}$ for the composition $\odot_{3}$. It follows that any isotropic sixth-order tensors can be decomposed into $\mathbb{A}=\mathbb{A}_{J}+\mathbb{A}_{K}$ where $\mathbb{A}_{J}=\mathbb{J} \odot_{3} \mathbb{A} \odot_{3} \mathbb{J}$ and $\mathbb{A}_{K}=\mathbb{K} \odot_{3} \mathbb{A} \odot_{3} \mathbb{K}$ and that the identity for the triple contraction is given by $\mathbb{I}=\mathbb{K}+\mathbb{J}$. This decomposition will be useful for providing various results and particularly for the product between two sixth-order tensors or the inversion of sixthorder tensors. Note that the decomposition $\left(\mathbb{K}_{n}, \mathbb{J}_{m}\right)$ gives rise to a generalization of the definition of the spherical and deviatoric parts of a third-order tensors, commonly used for two-order tensors, as it will be discussed in section 3 . The basis $\left(\mathbb{K}_{n}, \mathbb{J}_{m}\right)$ contains two tensors having the major symmetry, which are $\mathbb{K}_{1}$ and $\mathbb{K}_{2}$. Other tensors do not possess the major symmetry. The condition for tensor $\mathbb{A}$ having the major symmetry is:

$$
\left\{\begin{array}{l}
\alpha_{3}+2 \alpha_{5}=\alpha_{6}+2 \alpha_{4}, \quad \alpha_{7}+3 \alpha_{10}+\alpha_{13}=3 \alpha_{8}+\alpha_{11}+\alpha_{14} \\
\alpha_{8}+\alpha_{11}+3 \alpha_{14}=\alpha_{9}+3 \alpha_{12}+\alpha_{15}, \quad 3 \alpha_{9}+\alpha_{12}+\alpha_{15}=\alpha_{7}+\alpha_{10}+3 \alpha_{13}
\end{array}\right.
$$

Let us consider now a sixth-order tensor $\mathbb{A} \in \mathcal{E}_{6}$ and let us denote by $\alpha_{1}, \ldots, \alpha_{15}$ its components in the basis $\left(\mathbb{K}_{n}, \mathbb{J}_{m}\right)$, as in (7). Let us do the same with a second isotropic sixth-order tensor $\mathbb{B}$ and let us denote by $\beta_{1}, \ldots, \beta_{15}$ its components in $\left(\mathbb{K}_{n}, \mathbb{J}_{n}\right)$. We aim at computing the components of tensor $\mathbb{C}$ defined by $\mathbb{C}=\mathbb{B} \odot_{3} \mathbb{A}$. As explained above, any sixth-order tensor $\mathbb{C}$ can be decomposed along the two orthogonal subspaces $\mathcal{J}$ and $\mathcal{K}$ as follows: $\mathbb{C}=\mathbb{C}_{J}+\mathbb{C}_{K}$. It follows that $\mathbb{C}_{K}=\mathbb{B}_{K} \odot_{3} \mathbb{A}_{K}$ and $\mathbb{C}_{J}=\mathbb{B}_{J} \odot_{3} \mathbb{A}_{J}$. The computation of the components of $\mathbb{C}_{K}$ and $\mathbb{C}_{J}$ can be performed by using tables 1 and 2. We denote by $\gamma_{n}$ the components of $\mathbb{C}$ in the basis $\left(\mathbb{K}_{n}, \mathbb{J}_{n}\right)$. These components are given by:

$$
\left\{\begin{array}{l}
\gamma_{1}=\beta_{1} \alpha_{1}, \quad \gamma_{2}=\beta_{2} \alpha_{2}, \quad \gamma_{3}=\beta_{3} \alpha_{3}+\beta_{4} \alpha_{5}, \quad \gamma_{4}=\beta_{3} \alpha_{4}+\beta_{4} \alpha_{6} \\
\gamma_{5}=\beta_{5} \alpha_{3}+\beta_{6} \alpha_{5}, \quad \gamma_{6}=\beta_{5} \alpha_{4}+\beta_{6} \alpha_{6}, \quad \gamma_{7}=\beta_{7} \alpha_{7}+\beta_{8} \alpha_{10}+\beta_{9} \alpha_{13} \\
\gamma_{8}=\beta_{7} \alpha_{8}+\beta_{8} \alpha_{11}+\beta_{9} \alpha_{14}, \quad \gamma_{9}=\beta_{7} \alpha_{9}+\beta_{8} \alpha_{12}+\beta_{9} \alpha_{15} \\
\gamma_{10}=\beta_{10} \alpha_{7}+\beta_{11} \alpha_{10}+\beta_{12} \alpha_{13}, \quad \gamma_{11}=\beta_{10} \alpha_{8}+\beta_{11} \alpha_{11}+\beta_{12} \alpha_{14} \\
\gamma_{12}=\beta_{10} \alpha_{9}+\beta_{11} \alpha_{12}+\beta_{12} \alpha_{15}, \quad \gamma_{13}=\beta_{13} \alpha_{7}+\beta_{14} \alpha_{10}+\beta_{15} \alpha_{13} \\
\gamma_{14}=\beta_{13} \alpha_{8}+\beta_{14} \alpha_{11}+\beta_{15} \alpha_{14}, \quad \gamma_{15}=\beta_{13} \alpha_{9}+\beta_{14} \alpha_{12}+\beta_{15} \alpha_{15}
\end{array}\right.
$$

We now look at the inverse of tensor $\mathbb{A}$, namely we search $\mathbb{B}$ which verify the equation $\mathbb{B} \odot_{3} \mathbb{A}=\mathbb{A} \odot_{3} \mathbb{B}=\mathbb{I}$ where it is recalled that $\mathbb{I}=\mathbb{K}+\mathbb{J}$ is the identity for the triple contraction. Let us recall that the decomposition of $\mathbb{J}$ and $\mathbb{K}$ in the $\left(\mathbb{K}_{n}, \mathbb{J}_{m}\right)$ basis, reads $\mathbb{K}=\mathbb{K}_{1}+\mathbb{K}_{2}+\mathbb{K}_{3}+\mathbb{K}_{6}, \mathbb{J}=\mathbb{J}_{1}+\mathbb{J}_{5}+\mathbb{J}_{9}$. Consequently, the linear system giving the components of $\mathbb{B}$ can be obtained from (9) in which we put $\gamma_{1}=\gamma_{2}=\gamma_{3}=\gamma_{6}=\gamma_{7}=$ $\gamma_{11}=\gamma_{15}=1$ and all other $\gamma_{n}=0$. The computation of the $\beta_{n}$ for $n=1 . .15$ can be performed by a few simple algebraic equations. It leads to: 


$$
\left\{\begin{array}{l}
\beta_{1}=\frac{1}{\alpha_{1}}, \quad \beta_{2}=\frac{1}{\alpha_{2}}, \quad \beta_{3}=\frac{\alpha_{6}}{\Omega}, \quad \beta_{4}=-\frac{\alpha_{4}}{\Omega}, \quad \beta_{5}=-\frac{\alpha_{5}}{\Omega}, \quad \beta_{6}=\frac{\alpha_{3}}{\Omega} \\
\beta_{7}=\frac{\alpha_{12} \alpha_{14}-\alpha_{11} \alpha_{15}}{\Delta}, \beta_{8}=\frac{\alpha_{8} \alpha_{15}-\alpha_{9} \alpha_{14}}{\Delta}, \beta_{9}=\frac{\alpha_{9} \alpha_{11}-\alpha_{8} \alpha_{12}}{\Delta} \\
\beta_{10}=\frac{\alpha_{10} \alpha_{15}-\alpha_{12} \alpha_{13}}{\Delta}, \beta_{11}=\frac{\alpha_{9} \alpha_{13}-\alpha_{7} \alpha_{15}}{\Delta}, \beta_{12}=\frac{\alpha_{7} \alpha_{12}-\alpha_{9} \alpha_{10}}{\Delta} \\
\beta_{13}=\frac{\alpha_{11} \alpha_{13}-\alpha_{10} \alpha_{14}}{\Delta}, \beta_{14}=\frac{\alpha_{7} \alpha_{14}-\alpha_{8} \alpha_{13}}{\Delta}, \beta_{15}=\frac{\alpha_{8} \alpha_{10}-\alpha_{7} \alpha_{11}}{\Delta}
\end{array}\right.
$$

where $\Omega$ and $\Delta$ are given by:

$$
\begin{aligned}
& \Omega=\alpha_{3} \alpha_{6}-\alpha_{4} \alpha_{5} \\
& \Delta=\alpha_{7} \alpha_{12} \alpha_{14}+\alpha_{8} \alpha_{10} \alpha_{15}+\alpha_{9} \alpha_{11} \alpha_{13}-\alpha_{7} \alpha_{11} \alpha_{15}-\alpha_{8} \alpha_{12} \alpha_{13}-\alpha_{9} \alpha_{10} \alpha_{14}
\end{aligned}
$$

With the following condition for $\mathbb{A}$ having an inverse: $\alpha_{1} \alpha_{2} \Omega \Delta \neq 0$.

\section{Condition of positiveness of the elastic energy}

As it is well known from thermodynamic considerations, it is crucial to ensure that the elastic energy is positive definite ${ }^{1}$. Such a condition can be expressed easily for classically used isotropic second or fourth-order tensors, but it is not obvious to derive similar expressions for the case of higher degree elasticity. We now propose to determine the conditions of positiveness of the quadratic form $W(\boldsymbol{a})=\boldsymbol{a} \odot_{3} \mathbb{A} \odot_{3} \boldsymbol{a}=\boldsymbol{b} \odot_{3} \boldsymbol{a}$ where $\boldsymbol{b}=\boldsymbol{a} \odot_{3} \mathbb{A}$. Tensor $\mathbb{A}$ is now assumed to possess the major symmetry $\left(A_{i j k p q r}=A_{\text {pqrijk }}\right)$. As a consequence, its components in the basis $\left(\mathbb{K}_{n}, \mathbb{J}_{m}\right)$ comply to relations (8). Let us first introduce the spherical part of a third-order $\boldsymbol{a}$, denoted by $S(\boldsymbol{a})$, and the deviatoric part of $\boldsymbol{a}$, denoted by $D(\boldsymbol{a})$ :

$$
\begin{aligned}
& D(\boldsymbol{a})=\mathbb{K} \odot_{3} \boldsymbol{a}, \quad[D(\boldsymbol{a})]_{i j k}=a_{i j k}-\left[\boldsymbol{\theta}_{1}\right]_{i} \delta_{j k}-\left[\boldsymbol{\theta}_{2}\right]_{j} \delta_{i k}-\left[\boldsymbol{\theta}_{3}\right]_{k} \delta_{i j} \\
& S(\boldsymbol{a})=\mathbb{J} \odot_{3} \boldsymbol{a}, \quad[S(\boldsymbol{a})]_{i j k}=\left[\boldsymbol{\theta}_{1}\right]_{i} \delta_{j k}+\left[\boldsymbol{\theta}_{2}\right]_{j} \delta_{i k}+\left[\boldsymbol{\theta}_{3}\right]_{k} \delta_{i j}
\end{aligned}
$$

where the components of $\boldsymbol{\theta}_{n}$ for $n=1,2,3$ are given by:

$$
\begin{aligned}
& {\left[\boldsymbol{\theta}_{1}\right]_{i}=\frac{1}{10}\left[4 a_{i p p}-a_{p i p}-a_{p p i}\right], \quad\left[\boldsymbol{\theta}_{2}\right]_{i}=\frac{1}{10}\left[4 a_{p i p}-a_{i p p}-a_{p p i}\right]} \\
& {\left[\boldsymbol{\theta}_{3}\right]_{i}=\frac{1}{10}\left[4 a_{p p i}-a_{i p p}-a_{p i p}\right]}
\end{aligned}
$$

and it is recalled that $\mathbb{J}=\mathbb{J}_{1}+\mathbb{J}_{5}+\mathbb{J}_{9}$ and $\mathbb{K}=\mathbb{K}_{1}+\mathbb{K}_{2}+\mathbb{K}_{3}+\mathbb{K}_{6}$. Effecting the same operations with the third-order tensor $\boldsymbol{b}$, it appears that $W(\boldsymbol{a})$ can be decomposed into $W(\boldsymbol{a})=W_{D}(\boldsymbol{a})+W_{S}(\boldsymbol{a})$ where:

$$
W_{D}(\boldsymbol{a})=D(\boldsymbol{b}) \odot_{3} D(\boldsymbol{a}), \quad W_{S}(\boldsymbol{a})=S(\boldsymbol{b}) \odot_{3} S(\boldsymbol{a})
$$

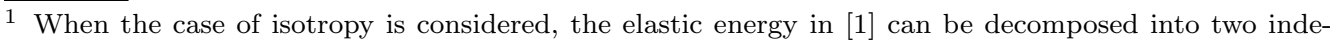
pendent terms corresponding to a two-order and a third-order tensor valued quadratic functions. Here we only focus our attention on the third-order valued quadratic function 
where $\boldsymbol{b}$ is given by (1). It follows that the positiveness of $W(\boldsymbol{a})$ is ensured if and only if $D(\boldsymbol{b}) \odot_{3} D(\boldsymbol{a}) \geq 0$ and $S(\boldsymbol{b}) \odot_{3} S(\boldsymbol{a}) \geq 0$. We now introduce the six deviatoric sub-parts of a third-order tensor $\boldsymbol{a}$, denoted by $D_{n}(\boldsymbol{a})=\mathbb{K}_{n} \odot_{3} \boldsymbol{a}$ for $n=1 . .6$, whose components are given by:

$$
\begin{aligned}
& {\left[D_{1}(\boldsymbol{a})\right]_{i j k}=\varepsilon_{i j k} \chi, \quad\left[D_{2}(\boldsymbol{a})\right]_{i j k}=\kappa_{i j k}, \quad\left[D_{3}(\boldsymbol{a})\right]_{i j k}=\varepsilon_{i j p}\left[\boldsymbol{\chi}_{1}\right]_{p k}} \\
& {\left[D_{4}(\boldsymbol{a})\right]_{i j k}=\varepsilon_{i j p}\left[\boldsymbol{\chi}_{2}\right]_{p k}, \quad\left[D_{5}(\boldsymbol{a})\right]_{i j k}=\varepsilon_{i k p}\left[\chi_{1}\right]_{p j}, \quad\left[D_{6}(\boldsymbol{a})\right]_{i j k}=\varepsilon_{i k p}\left[\chi_{2}\right]_{p j}}
\end{aligned}
$$

where $\varepsilon_{i j k}$ is the permutation symbol. The scalar $\chi$ and tensors $\chi_{1}, \chi_{2}, \kappa$ are given by:

$$
\begin{aligned}
& \kappa_{i j k}=\frac{1}{6}\left\{[D(\boldsymbol{a})]_{i j k}+[D(\boldsymbol{a})]_{k i j}+[D(\boldsymbol{a})]_{j k i}+[D(\boldsymbol{a})]_{k j i}+[D(\boldsymbol{a})]_{i k j}+[D(\boldsymbol{a})]_{j i k}\right\} \\
& \chi=\frac{1}{6} \varepsilon_{i j k}[D(\boldsymbol{a})]_{i j k} \\
& {\left[\chi_{1}\right]_{i j}=\frac{1}{6} \varepsilon_{i p q}\left([D(\boldsymbol{a})]_{p q j}+[D(\boldsymbol{a})]_{j q p}\right)+\frac{1}{6} \varepsilon_{j p q}\left([D(\boldsymbol{a})]_{p q i}+[D(\boldsymbol{a})]_{i q p}\right)} \\
& {\left[\chi_{2}\right]_{i j}=\frac{1}{6} \varepsilon_{i p q}\left([D(\boldsymbol{a})]_{j p q}+[D(\boldsymbol{a})]_{p j q}\right)+\frac{1}{6} \varepsilon_{j p q}\left([D(\boldsymbol{a})]_{i p q}+[D(\boldsymbol{a})]_{p i q}\right)}
\end{aligned}
$$

$\chi_{1}$ and $\chi_{2}$ are two symmetric, traceless, second-order tensors whereas $\boldsymbol{\kappa}$ is a symmetric traceless third-order tensor (namely $\kappa_{i j k}$ is invariant by any permutation of its indices $i, j, k$ and $\left.\kappa_{i j j}=\kappa_{i j i}=\kappa_{j j i}=0\right)$. Tensors $\chi_{1}, \chi_{2}, \kappa$ are respectively defined by 5,5 and 7 independent coefficients. Similarly, the triple contraction between the $\mathbb{J}_{n}$ and $\boldsymbol{a}$ produces the 9 spherical sub-parts of $\boldsymbol{a}$, denoted $S_{n}(\boldsymbol{a})=\mathbb{J}_{n} \odot_{3} \boldsymbol{a}$. Their components are given by:

$$
\begin{aligned}
& {\left[S_{1}(\boldsymbol{a})\right]_{i j k}=\left[\boldsymbol{\theta}_{1}\right]_{i} \delta_{j k}, \quad\left[S_{2}(\boldsymbol{a})\right]_{i j k}=\left[\boldsymbol{\theta}_{2}\right]_{i} \delta_{j k}, \quad\left[S_{3}(\boldsymbol{a})\right]_{i j k}=\left[\boldsymbol{\theta}_{3}\right]_{i} \delta_{j k}} \\
& {\left[S_{4}(\boldsymbol{a})\right]_{i j k}=\left[\boldsymbol{\theta}_{1}\right]_{j} \delta_{i k}, \quad\left[S_{5}(\boldsymbol{a})\right]_{i j k}=\left[\boldsymbol{\theta}_{2}\right]_{j} \delta_{i k}, \quad\left[S_{6}(\boldsymbol{a})\right]_{i j k}=\left[\boldsymbol{\theta}_{3}\right]_{j} \delta_{i k}} \\
& {\left[S_{7}(\boldsymbol{a})\right]_{i j k}=\left[\boldsymbol{\theta}_{1}\right]_{k} \delta_{i j}, \quad\left[S_{8}(\boldsymbol{a})\right]_{i j k}=\left[\boldsymbol{\theta}_{2}\right]_{k} \delta_{i j}, \quad\left[S_{9}(\boldsymbol{a})\right]_{i j k}=\left[\boldsymbol{\theta}_{3}\right]_{k} \delta_{i j}}
\end{aligned}
$$

Where $\boldsymbol{\theta}_{1}, \boldsymbol{\theta}_{2}, \boldsymbol{\theta}_{3}$ are given by (13).

As quoted in the previous section, $\mathbb{I}$ is the identity for the composition $\odot_{3}$, namely: $\boldsymbol{a}=\mathbb{I} \odot_{3} \boldsymbol{a}$. Since the decomposition of $\mathbb{I}$ in the basis $\left(\mathbb{K}_{n}, \mathbb{J}_{m}\right)$ is $\mathbb{I}=\mathbb{K}_{1}+\mathbb{K}_{2}+\mathbb{K}_{3}+$ $\mathbb{K}_{6}+\mathbb{J}_{1}+\mathbb{J}_{5}+\mathbb{J}_{9}$, it follows that any third-order tensor $\boldsymbol{a}$ can be decomposed into:

$$
\boldsymbol{a}=\underbrace{D_{1}(\boldsymbol{a})+D_{2}(\boldsymbol{a})+D_{3}(\boldsymbol{a})+D_{6}(\boldsymbol{a})}_{=D(\boldsymbol{a})}+\underbrace{S_{1}(\boldsymbol{a})+S_{5}(\boldsymbol{a})+S_{9}(\boldsymbol{a})}_{=S(\boldsymbol{a})}
$$

Note that $\boldsymbol{a}$ is defined by 27 independents components whereas tensors $D_{1}(\boldsymbol{a}), D_{2}(\boldsymbol{a})$, $D_{3}(\boldsymbol{a}), D_{6}(\boldsymbol{a}), S_{1}(\boldsymbol{a}), S_{5}(\boldsymbol{a}), S_{9}(\boldsymbol{a})$ are respectively defined by $1,7,5,5,3,3,3$ independents components. The decomposition (18) can be also applied for $\boldsymbol{b}$ and the linear relation (1) can then be put into the form: 


$$
\left\{\begin{array}{l}
D_{1}(\boldsymbol{b})=\alpha_{1} D_{1}(\boldsymbol{a}) \\
D_{2}(\boldsymbol{b})=\alpha_{2} D_{1}(\boldsymbol{a}) \\
D_{3}(\boldsymbol{b})=\alpha_{3} D_{3}(\boldsymbol{a})+\alpha_{4} D_{4}(\boldsymbol{a}) \\
D_{6}(\boldsymbol{b})=\alpha_{5} D_{5}(\boldsymbol{a})+\alpha_{6} D_{6}(\boldsymbol{a}) \\
S_{1}(\boldsymbol{b})=\alpha_{7} S_{1}(\boldsymbol{a})+\alpha_{8} S_{2}(\boldsymbol{a})+\alpha_{9} S_{3}(\boldsymbol{a}) \\
S_{5}(\boldsymbol{b})=\alpha_{10} S_{4}(\boldsymbol{a})+\alpha_{11} S_{5}(\boldsymbol{a})+\alpha_{12} S_{6}(\boldsymbol{a}) \\
S_{9}(\boldsymbol{b})=\alpha_{13} S_{7}(\boldsymbol{a})+\alpha_{14} S_{8}(\boldsymbol{a})+\alpha_{15} S_{9}(\boldsymbol{a})
\end{array}\right.
$$

Again, table 1 and 2 must be used to derive expressions above.

Taking into account (18), (19) with definitions (15) and (17), it follows that:

$$
\begin{aligned}
W_{D}(\boldsymbol{a})= & 6 \alpha_{1} \chi^{2}+\alpha_{2} \boldsymbol{\kappa} \odot_{3} \boldsymbol{\kappa}+\varphi_{1} \boldsymbol{\chi}_{1} \odot_{2} \boldsymbol{\chi}_{1}+\varphi_{2} \boldsymbol{\chi}_{2} \odot_{2} \boldsymbol{\chi}_{2}+2 \varphi_{3} \boldsymbol{\chi}_{1} \odot_{2} \boldsymbol{\chi}_{2} \\
W_{S}(\boldsymbol{a})= & \psi_{1} \boldsymbol{\theta}_{1} \odot_{1} \boldsymbol{\theta}_{1}+\psi_{2} \boldsymbol{\theta}_{2} \odot_{1} \boldsymbol{\theta}_{2}+\psi_{3} \boldsymbol{\theta}_{3} \odot_{1} \boldsymbol{\theta}_{3}+2 \psi_{4} \boldsymbol{\theta}_{2} \odot_{1} \boldsymbol{\theta}_{3} \\
& +2 \psi_{5} \boldsymbol{\theta}_{1} \odot_{1} \boldsymbol{\theta}_{3}+2 \psi_{6} \boldsymbol{\theta}_{1} \odot_{1} \boldsymbol{\theta}_{2}
\end{aligned}
$$

Where coefficients $\varphi_{n}$ and $\psi_{n}$ are given by:

$$
\begin{aligned}
& \varphi_{1}=2 \alpha_{3}+\alpha_{5}, \quad \varphi_{2}=\alpha_{4}+2 \alpha_{6}, \quad \varphi_{3}=\alpha_{3}+2 \alpha_{5}=2 \alpha_{4}+\alpha_{6} \\
& \psi_{1}=3 \alpha_{7}+\alpha_{10}+\alpha_{13}, \quad \psi_{2}=\alpha_{8}+3 \alpha_{11}+\alpha_{14}, \quad \psi_{3}=\alpha_{9}+\alpha_{12}+3 \alpha_{15} \\
& \psi_{4}=\alpha_{8}+\alpha_{11}+3 \alpha_{14}=\alpha_{9}+3 \alpha_{12}+\alpha_{15} \\
& \psi_{5}=3 \alpha_{9}+\alpha_{12}+\alpha_{15}=\alpha_{7}+\alpha_{10}+3 \alpha_{13} \\
& \psi_{6}=\alpha_{7}+3 \alpha_{10}+\alpha_{13}=3 \alpha_{8}+\alpha_{11}+\alpha_{14}
\end{aligned}
$$

The condition of positiveness of the elastic potential is then given by:

$$
\alpha_{1} \geq 0, \quad \alpha_{2} \geq 0, \quad \varphi_{1} \geq 0, \quad \varphi_{2} \geq 0, \quad \Psi_{1} \geq 0, \quad \Psi_{2} \geq 0, \quad \Psi_{3} \geq 0
$$

where $\Psi_{1}, \Psi_{2}, \Psi_{3}$ are the eigenvalues of the $3 \times 3$ matrix:

$$
\Psi=\left(\begin{array}{lll}
\psi_{1} & \psi_{6} & \psi_{5} \\
\psi_{6} & \psi_{2} & \psi_{4} \\
\psi_{5} & \psi_{4} & \psi_{3}
\end{array}\right)
$$

\section{Conclusion}

In the present paper, we provide an irreducible basis for sixth-order isotropic tensors. This basis is constituted of 15 elements denoted $\mathbb{K}_{n}$ for $n=1,6$ and $\mathbb{J}_{n}$ for $n=1,9$ and it appears to be useful for doing the classical tensorial operations. For instance, a 
closed form expression of the inverse of an isotropic sixth-order tensor has been derived in section 3. Moreover, the projection of a third-order tensor along the tensors of the new basis $\left(\mathbb{K}_{n}, \mathbb{J}_{m}\right)$, gives rise to a generalization of a spherical and deviatoric parts commonly used for two-order tensors to the case of third-order tensors. This decomposition has been subsequently used for deriving the condition of positiveness of the quadratic form $\boldsymbol{a} \odot_{3}$ $\mathbb{A} \odot_{3} \boldsymbol{a}$. For instance, this last result can be used for giving the condition of positiveness of the elastic energy of microstructured elastic media.

\section{References}

[1] R.D. Mindlin. Micro-structure in linear elasticity. Archive for Rational Mechanics and Analysis. Vol. 16, pp. 51-78, 1964

[2] R.D. Mindlin, N.N. Eshel . On first strain gradients theory in linear elasticity. International Journal of Solids and Structures. Vol. 4, pp. 109-124, 1968.

[3] V. Monchiet, G. Bonnet. Inversion of higher order isotropic tensors with minor symmetries and solution of higher order heterogeneity problems. Proc. Roy. Soc. A. In press.

[4] S. Pennisi, M. Trovato. On the irreducibility of Professor G.F. Smith's representations for isotropic functions, Int. J. Engng. Sci. Vol. 25, pp. 1056-1065, 1987.

[5] S. Pennisi. On third order tensor-valued isotropic functions. Int. J. Engng. Sci. Vol. 30, pp. 879-892, 1992.

[6] G.F. Smith, B.A. Younis. Isotropic tensor-valued polynomial function of second and third-order tensors. Int. J. Engng. Sci. Vol. 43, pp. 447-456, 2005.

[7] V.P. Smyshlyaev, N.A. Fleck The role of strain gradients in the grain size effect for polycrystals. J. Mech. Phys. Solids. Vol. 44, pp. 465-496, 1996.

[8] R. Toupin. Elastic materials with couple-stresses. Archive for Rational Mechanics and Analysis. Vol. 11, Issue 1, pp. 385-414, 1962.

\section{Appendix A. Appendix : Base change relations}

The components $\alpha_{i}$ of a $6^{\text {th }}$ order tensor along the basis $\left(\mathbb{J}_{n}, \mathbb{K}_{m}\right)$ from its components $a_{j}$ along the bases $\left(T_{n}\right)$ are given by:

$$
\begin{aligned}
& \alpha_{1}=a_{1}+a_{2}+a_{3}-a_{4}-a_{5}-a_{6} ; \quad \alpha_{2}=a_{1}+a_{2}+a_{3}+a_{4}+a_{5}+a_{6} \\
& \alpha_{3}=a_{1}-a_{2}+a_{4}-a_{6} ; \quad \alpha_{4}=-a_{2}+a_{3}+a_{5}-a_{6} \\
& \alpha_{5}=a_{2}-a_{3}-a_{4}+a_{5} ; \quad \alpha_{6}=a_{1}-a_{3}+-a_{4}+a_{6} \\
& \alpha_{7}=a_{1}+a_{5}+a_{13}+3 a_{14}+a_{15} ; \quad \alpha_{8}=a_{3}+a_{6}+a_{13}+a_{14}+3 a_{15} \\
& \alpha_{9}=a_{2}+a_{4}+3 a_{13}+a_{14}+a_{15} ; \quad \alpha_{10}=a_{2}+a_{6}+a_{10}+3 a_{11}+a_{12} \\
& \alpha_{11}=a_{1}+a_{4}+a_{10}+a_{11}+3 a_{12} ; \quad \alpha_{12}=a_{3}+a_{5}+3 a_{10}+a_{11}+a_{12} \\
& \alpha_{13}=a_{3}+a_{4}+a_{7}+3 a_{8}+a_{9} ; \quad \alpha_{14}=a_{2}+a_{5}+a_{7}+a_{8}+3 a_{9} \\
& \alpha_{15}=a_{1}+a_{6}+3 a_{7}+a_{8}+a_{9}
\end{aligned}
$$

